\title{
Estimation of Time-Varying Multipath Rayleigh Fading Channels in Asynchronous DS-CDMA Systems
}

\author{
Pei Xiao ${ }^{1}$, Erik Ström ${ }^{2}$, Rolando Carrasco $^{1}$ \\ ${ }^{1}$ School of Computing and Information Technology \\ University of Wolverhampton, WV1, 4QL, United Kingdom \\ E-mail: pei.xiao@wlv.ac.uk, r.carrasco@wlv.ac.uk \\ ${ }^{2}$ Communication Systems Group, Dept. of Signals and Systems \\ Chalmers University of Technology, SE-412 96, Göteborg, Sweden \\ E-mail: erik.strom@s2.chalmers.se
}

\begin{abstract}
In this paper, we present channel estimation algorithms for the asynchronous directsequence code-division multiple access (DS-CDMA) systems employing orthogonal signalling formats and long scrambling codes. The performance of a communication system depends largely on its ability to retrieve an accurate measurement of the underlying channel. We investigated channel estimation algorithms under different conditions. The estimated channel information is used to enable coherent data detection to combat the detrimental effect of multipath propagation of the transmitted signal as well as multiple access interference (MAI). Different channel estimation schemes are evaluated and compared in terms of mean square error (MSE) of the channel estimate and the bit error rate (BER) performance. Based on our analysis and numerical results, some recommendations are made on how to choose appropriate channel estimators in practical systems.
\end{abstract}

\section{INTRODUCTION}

Multiuser detection (MUD) techniques are widely used to combat the detrimental effects of multipath fading and MAI, which are the major impairments in CDMA communication systems. Most multiuser detectors rely on accurate channel information, which needs to be estimated in practice. If the channel estimates are not reliable, the performance of algorithms such as multiuser detectors degrade significantly. Channel estimation consequently is an important issue in mobile communications and good channel estimates have a very important impact on the overall performance of the system.

The estimation of channel parameters in a DS-CDMA system with orthogonal signalling formats has been the subject of study in several papers (see, for instance, [1], [2], $[3],[4])$. The maximum likelihood (ML) channel estimator for long code CDMA systems over time-varying multipath channels was employed in [1]. It was shown in [2], [3] that the channel parameters can be estimated with a maximum correlator output. In [4], a blind channel estimation strategy based on an adaptive Wiener filtering approach that yields unbiased channel estimates and low estimation variance for CDMA system using random codes was proposed.

The purpose of this paper is to make an extensive investigation on different alternatives for estimating timevarying multipath Rayleigh fading channels. We take an integrated approach such that channel estimation is coupled with data detection (interference cancellation in particular). The maximum likelihood (ML) algorithm is shown to be efficient for estimating multipath Rayleigh fading channels when the underlying channels are completely unknown and when the fading processes are slow. With the knowledge of the noise and channel statistics, we can employ linear MMSE estimator to yield more noise-resistant estimate. Other alternatives, like the first and second order Kalman filters which take into account the correlative nature of the Rayleigh fading channel are also introduced.

\section{System Model}

The system under study is an asynchronous DS-CDMA system with orthogonal modulation. The transmitted chip sequence from a particular user is the concatenation of one of $M$ possible Walsh sequences (representing the transmitted symbol) and a long scrambling code. Fig. 1 shows the signal path for the $k^{t h}$ user. The $j^{\text {th }}$ block of $\log _{2} M$ information bits denoted by $\mathbf{u}_{k}(j) \in\{+1,-1\}^{\log _{2} M}$ is mapped into $\mathbf{w}_{k}(j) \in\left\{\mathbf{w}_{0}, \cdots, \mathbf{w}_{M-1}\right\}$, which is one of the $M$ orthogonal signal alternatives. The Walsh codeword $\mathbf{w}_{k}(j) \in\{+1,-1\}^{M}$, is then repetition encoded into $\mathbf{s}_{k}(j) \in\{+1,-1\}^{N}$ so that each bit of the Walsh codeword is spread (repetition coded) into $N_{c}=N / M$ chips, and each Walsh symbol is represented by $N$ chips. The Walsh sequence $\mathbf{s}_{k}(j)$ is then scrambled (randomized) by a scrambling code unique to each user to form the transmitted chip sequence $\mathbf{a}_{k}(j)=\mathbf{C}_{k}(j) \mathbf{s}_{k}(j) \in\{+1,-1\}^{N}$ where $\mathbf{C}_{k}(j) \in\{-1,0,+1\}^{N \times N}$ is a diagonal matrix whose diagonal elements correspond to the scrambling code for the $k^{\text {th }}$ user's $j^{\text {th }}$ symbol. The purpose of scrambling is to separate users. In this paper, we focus on the use of long codes, e.g., the scrambling code differs from symbol to symbol.

The baseband signal is formed by pulse amplitude modulating $\mathbf{a}_{k}(j)$ with a unit-energy rectangular chip waveform $\psi(t)$. The baseband signal is then multiplied with a carrier 


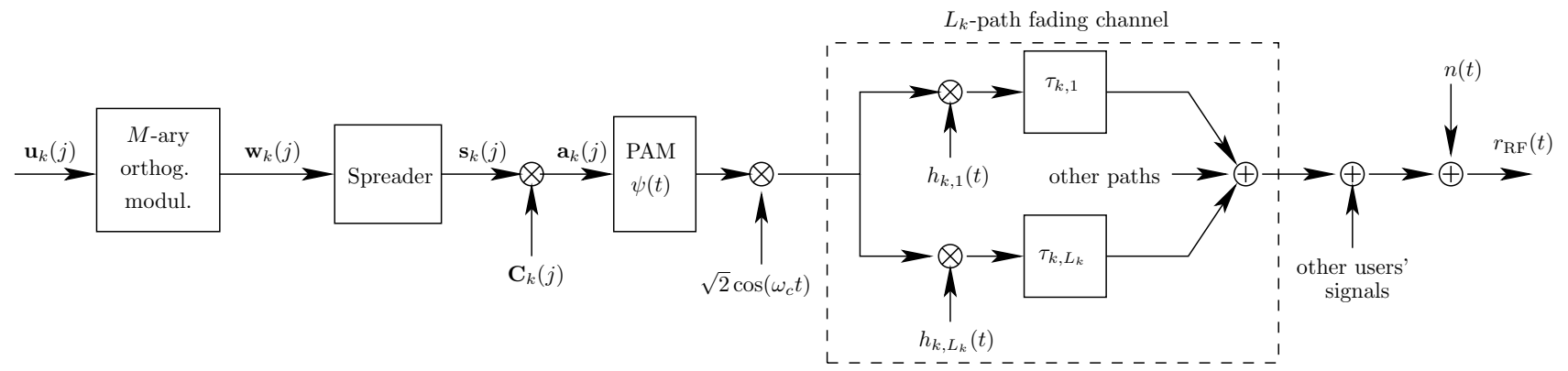

Fig. 1. Block diagram of the transmitter.

and transmitted over a Rayleigh fading channel with $L_{k}$ resolvable paths, having time-varying complex channel gains $h_{k, 1}(t), h_{k, 2}(t), \ldots, h_{k, L_{k}}(t)$ and delays $\tau_{k, 1}, \tau_{k, 2}, \ldots, \tau_{k, L_{k}}$. The received signal is the sum of $K$ users' signals plus additive white complex Gaussian noise $n(t)$. The received vector corresponding to the $k^{\text {th }}$ user's $j^{\text {th }}$ symbol after frequency down-conversion and chip matched filtering is denoted as $\mathbf{r}(k, j)$ and can be written in vector form as

$$
\begin{aligned}
& \mathbf{r}(k, j)=\mathbf{A}(k, j) \mathbf{h}(j)+\mathbf{n}(k, j) \\
& =\mathbf{X}_{k}(j) \mathbf{h}_{k}(j)+\operatorname{ISI}(\mathrm{k}, \mathrm{j})+\operatorname{MAI}(\mathrm{k}, \mathrm{j})+\mathbf{n}(\mathrm{k}, \mathrm{j})
\end{aligned}
$$

where the columns of the matrix $\mathbf{A}(k, j)$ are delayed versions of the transmitted chip sequences $\mathbf{a}_{k}(j)$ for $k=$ $1,2, \cdots, K$, one column per path. The columns are weighted together by $\mathbf{h}(j)$, whose elements are the path gains of all users' paths. The received vector $\mathbf{r}(k, j)$ can be written as the sum of four terms: the signal of interest $\mathbf{X}_{k}(j) \mathbf{h}_{k}(j)$, the intersymbol interference (ISI), the multiple access interference (MAI), and the noise represented by $\mathbf{n}(k, j)$, which is a vector of complex Gaussian noise samples with zero mean and variance $N_{0}$. The columns of the matrix $\mathbf{X}_{k}(j)$ are essentially shifted versions of the chips due to the $k^{\text {th }}$ user's $j^{\text {th }}$ symbol, one column per path (the shift is determined by the path delay). The vector

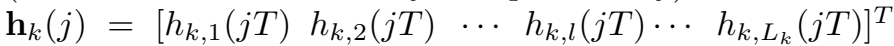
corresponds to the channel gains of the $k^{\text {th }}$ user's paths, it is a part of $\mathbf{h}(j)$.

The task of the demodulator is to detect the information bits from all users, i.e., detect $\mathbf{u}_{k}(j)$ or equivalently, the transmitted Walsh codes $\mathbf{w}_{k}(j)$ for $k=1,2, \ldots, K, j=$ $1,2, \ldots, L_{b}$ ( $L_{b}$ is the block length) given the observation $\mathbf{r}(k, j)$.

With conventional matched filter $(\mathrm{MF})$, the decision is formed by correlating the received signal with the $M$ possible transmitted waveforms. This simple scheme is particularly useful in the beginning of the detection process when the estimates of the fading channel are lacking, we must therefore carry out the detection in a noncoherent manner. However, it has poor performance in multiuser environment since it considers MAI as additive noise and the knowledge about MAI is not exploited in any way. An effective tool to increase the capacity of interference-limited CDMA systems is MUD, a method of jointly detecting all the users in the system. Among different MUD techniques, the interference cancellation schemes are known to be simple and effective for mitigation of MAI in long-code DS-CDMA systems. For the purpose of this study, we consider the multistage parallel interference cancellation (PIC) scheme introduced in [1].

\section{Estimation of Fading Processes}

The task of a channel estimator is to estimate the fading vector $\mathbf{h}$ in equation (1) given the received observation $\mathbf{r}$ and the transmitted data. The purpose of channel estimation is to enable $M$-ary orthogonal signals to be demodulated coherently and a RAKE receiver to use a maximum ratio combining (MRC) scheme. Depending on the form of the data that can be retrieved, channel estimation can be either decision directed or pilot aided. In this paper, all the channel estimators are decision-directed and can work in conjunction with the coherent data detection. The estimation procedure uses the data estimates from the previous stage.

Depending on whether prior knowledge about the parameters to be estimated is used, statistical estimation can be conducted either with classical approach or Bayesian approach. Both methods are discussed in this paper. The maximum likelihood channel estimator is derived based on the classical approach. In this case, the channel vector $\mathbf{h}$ is assumed to be deterministic and unknown. While with a Bayesian approach, $\mathbf{h}$ is assumed to be a random vector whose particular realization needs to be estimated. It improves the estimation accuracy by exploiting some prior knowledge about $\mathbf{h}$. The Bayesian philosophy leads to the linear MMSE estimator and the Kalman filter for channel estimation. Those channel estimation algorithms will be presented below.

\section{A. Maximum Likelihood Channel Estimator}

Given an estimate of the data matrix $\mathbf{A}$ in (1), then $\mathbf{h}$ can be computed as $\hat{\mathbf{h}}=\hat{\mathbf{A}}^{\dagger} \mathbf{r}[1]$. The matrix $\hat{\mathbf{A}}^{\dagger}=\left(\hat{\mathbf{A}}^{*} \hat{\mathbf{A}}\right)^{-1} \hat{\mathbf{A}}^{*}$ denotes the left pseudoinverse of $\hat{\mathbf{A}}$ (assuming $\mathbf{A}$ has full column rank), and $\hat{\mathbf{A}}$ is an estimate of $\mathbf{A}$. In case of correct decisions, $\hat{\mathbf{A}}=\mathbf{A}$ and $\hat{\mathbf{h}}=\mathbf{A}^{\dagger} \mathbf{r}=\mathbf{A}^{\dagger}(\mathbf{A} \mathbf{h}+\mathbf{n})=\mathbf{h}+\mathbf{A}^{\dagger} \mathbf{n}$, which is an unbiased estimate of $\mathbf{h}$.

This procedure will suffer from a so-called dimensionality 
problem. When the total number of paths of all the users is greater than the number of chips in the vector $\mathbf{r}(k, j)$, i.e., $L_{\text {tot }} \geq N_{k}\left(L_{\text {tot }}=\sum_{k=1}^{K} L_{k}\right)$, the matrix $\hat{\mathbf{A}}$ will not have full column rank and the above mentioned procedure will become useless. The problem can be resolved by stacking several $\mathbf{r}(j)$ vectors on top of each other and assume the channel remains static during several symbol intervals. In particular, suppose $\mathbf{h}(j) \approx \mathbf{h}(j+1)$, the ML channel estimation algorithm using detected data $\hat{\mathbf{A}}$ can be reformulated as

$$
\hat{\mathbf{h}}^{\mathrm{ML}}(j)=\left[\begin{array}{c}
\hat{\mathbf{A}}(j) \\
\hat{\mathbf{A}}(j+1)
\end{array}\right]^{\dagger}\left[\begin{array}{c}
\mathbf{r}(j) \\
\mathbf{r}(j+1)
\end{array}\right],
$$

which will produce usable estimates as long as $2 N_{k}>L_{\text {tot }}$. Obviously, this scheme can be extended further by stacking several $\mathbf{r}(k, j)$ vectors on top of each other. The stacking also has the effect of noise averaging and tends to reduce the error of the channel estimation. However, for relatively fast fading channels, the stacking may have opposite effect and reduce the quality of the estimates.

\section{B. Linear MMSE Channel Estimator}

The linear MMSE algorithm for channel estimation computes a matrix $\mathbf{W}$, which is chosen to minimize the mean square error $E\left[\left\|\mathbf{h}-\mathbf{W}^{*} \mathbf{r}\right\|^{2}\right]$. The optimum matrix of $\mathbf{W}$ under the MMSE criterion can be computed as

$$
\begin{aligned}
\mathbf{W}_{\mathrm{MMSE}} & =\arg \min _{\mathbf{W}} \mathrm{E}\left[\left\|\mathbf{h}-\mathbf{W}^{*} \mathbf{r}\right\|^{2}\right]=\mathbf{R}^{-1} \mathbf{\Phi} \\
\mathbf{R} & =\mathrm{E}\left[\mathbf{r r}^{*}\right]=\mathrm{E}\left[(\mathbf{A h}+\mathbf{n})(\mathbf{A} \mathbf{h}+\mathbf{n})^{*}\right] \\
& =\mathbf{A} \mathbf{P} \mathbf{A}^{*}+N_{0} \mathbf{I} \\
\mathbf{\Phi} & =\mathrm{E}\left[\mathbf{r h}^{*}\right]=\mathrm{E}\left[(\mathbf{A} \mathbf{h}+\mathbf{n}) \mathbf{h}^{*}\right]=\mathbf{A P} \\
\mathbf{P} & =\mathrm{E}\left[\mathbf{h h}^{*}\right]=\operatorname{diag}\left(P_{1,1}, \cdots, P_{k, l}, \cdots, P_{K, L_{K}}\right)
\end{aligned}
$$

where $P_{k, l}$ is the average received power from the $k^{t h}$ user's $l^{\text {th }}$ path. Combining the above equations, the linear MMSE estimate of $\mathbf{h}$ can be formulated as

$$
\begin{aligned}
\hat{\mathbf{h}}^{\mathrm{LMMSE}} & =\mathbf{W}_{\mathrm{MMSE}}^{*} \mathbf{r}=\boldsymbol{\Phi}^{*} \mathbf{R}^{-1} \mathbf{r} \\
& =\mathbf{P}^{*} \hat{\mathbf{A}}^{*}\left(\hat{\mathbf{A}} \mathbf{P} \hat{\mathbf{A}}^{*}+N_{0} \mathbf{I}\right)^{-1} \mathbf{r}
\end{aligned}
$$

From (3), we see that the signal and noise power level $P_{k, l}$ and $N_{0}$ must be known or estimated to carry out LMMSE channel estimation. It is worth noticing that $\mathbf{A}$ does not need to be full rank to ensure the invertibility of $\left(\mathbf{A P A}^{*}+\right.$ $\left.N_{0} \mathbf{I}\right)$.

The fading processes are lowpass in nature with bandwidths that are determined by the Doppler frequency. We should therefore be able to improve the estimates by lowpass filtering (smoothing). A simple smoothing procedure is to feed $\hat{\mathbf{h}}(j)$ through an FIR filter with impulse response $g(n)$ [1], which yields the smoothed channel gain vector $\overline{\mathbf{h}}(j)$ as

$$
\overline{\mathbf{h}}(j)=\sum_{k=j-N_{s}}^{j+N_{s}} \hat{\mathbf{h}}(k) g(j-k)
$$

In our simulations, we use a smoothing filter derived from a Hamming window of length $2 N_{s}+1=19$ or 9 (for slow or fast fading channel), normalized such that $\sum_{k=-N_{s}}^{N_{s}} g(k)=$ 1. As will be evidenced by numerical results, smoothing operation really improves the results of ML and LMMSE channel estimates, and subsequently leads to more reliable data detection.

\section{Kalman Filter}

If $\mathbf{h}$ and its statistics are completely unknown, the ML estimator is the best we can do. However, we know that channel gains are correlated in time. One way to exploit this knowledge is to use smoothing filter to improve estimation results as introduced above. An alternative approach is to consider $\mathbf{h}$ to be a realization of a random process to be estimated in the development of channel estimation algorithm, which leads to the use of, e.g., a Kalman filter. The Kalman filter is a time-varying linear filter widely used in statistical estimation when the unknown parameters evolve in time according to some dynamic model [5]. It has been used, e.g., in [6], [7], [8], [9] for the purpose of channel estimation. Here, we extend the use of Kalman filter to estimation of multipath fading channels for orthogonal modulated CDMA systems.

We assume the channels are independently Rayleigh fading channels with the Clarke's power spectral density given by

$$
S(f)= \begin{cases}\frac{K}{\sqrt{\left(1-\left(f / f_{d}\right)^{2}\right.}}, & |f|<f_{d} \\ 0, & |f| \geq f_{d}\end{cases}
$$

where $f_{d}$ is the maximum Doppler frequency. The channel gain $h_{k, l}(t)$ is a complex circular Gaussian process with autocorrelation function $\mathrm{E}\left[h_{k, l}^{*}(t) h_{k, l}(t+\tau)\right]=P_{k, l} J_{0}\left(2 \pi f_{d} \tau\right)$ and $J_{0}(x)$ is the zeroth order Bessel function of the first kind.

The power spectral density expressed in (4) can be approximated by a finite order autoregressive (AR) process. The correlation between channel coefficients can simply be approximated by the following first-order vector GaussMarkov model as

$$
\mathbf{h}(j)=\mathbf{F h}(j-1)+\mathbf{u}(j)
$$

where $\mathbf{F} \in \mathbb{R}^{L_{\text {tot }} \times L_{\text {tot }}}$ is the state transition matrix and $\mathbf{u}(j) \in \mathbb{C}^{L_{\text {tot }}}$ is the driving noise vector (WGN sequence) with $\mathrm{E}[\mathbf{u}(j)]=\mathbf{0}$ and covariance matrix $\mathbf{Q}$. The current input $\mathbf{h}(j)$ depends only on the state of the system at the previous symbol time $\mathbf{h}(j-1)$ and the current input $\mathbf{u}(j)$. The state $\mathbf{h}(j-1)$ accumulates the effect of all past inputs to the system.

Since different paths are uncorrelated with each other, we can let $\mathbf{F}$ and $\mathbf{Q}$ be diagonal matrices, i.e., $\mathbf{F}=\operatorname{diag}\left(f_{1,1}, f_{1,2}, \ldots, f_{k, l}, \ldots, f_{K, L_{K}}\right)$ and $\mathbf{Q}=$ $\operatorname{diag}\left(\sigma_{1,1}^{2}, \sigma_{1,2}^{2}, \ldots, \sigma_{k, l}^{2}, \ldots, \sigma_{K, L_{K}}^{2}\right)$. Using the notation $h_{k, l}[j]=h_{k, l}(j T)$, the vector Gauss-Markov model becomes 
$L_{\text {tot }}$ independent scalar models: $h_{k, l}[j]=f_{k, l} h_{k, l}[j-1]+$ $u_{k, l}[j]$. By solving the equations

$$
\begin{gathered}
\mathrm{E}\left\{h_{k, l}[j] h_{k, l}^{*}[j-1]\right\} \\
\begin{array}{rl}
\mathrm{E}\left\{\left|h_{k, l}[j]\right|^{2}\right\}=f_{k, l}^{2} & \mathrm{E}\left\{\left|h_{k, l}[j-1]\right|^{2}\right\}+\mathrm{E}\left\{\left|u_{k, l}[j]\right|^{2}\right\} \\
& =f_{k, l}^{2} P_{k, l}+\sigma_{k, l}^{2}
\end{array}
\end{gathered}
$$

we obtain the coefficients for the first-order model

$$
\begin{aligned}
f_{k, l} & =\mathrm{E}\left\{h_{k, l}^{*}[j] h_{k, l}[j-1]\right\} / P_{k, l}=J_{0}\left(2 \pi f_{d} T\right) \\
\sigma_{k, l}^{2} & =P_{k, l}\left(1-f_{k, l}^{2}\right)
\end{aligned}
$$

Note that the maximum Doppler frequency $f_{d}$ can differ from path to path. As a special case, when all the paths from different users have the same received power and Doppler frequency, the matrices $\mathbf{F}, \mathbf{Q}$ are simplified to $\mathbf{F}=f \mathbf{I}, \mathbf{Q}=\sigma_{Q}^{2} \mathbf{I}$. Some training method was proposed in [6] to estimate the state transition matrix $\mathbf{F}$, also the algorithm for tracking Doppler shift is addressed in [8]. Here we focus on the estimation of complex channel gains and assume that the Doppler shift is known after a training phase.

Recall that our observation vector (measurement model) is, $\mathbf{r}(j)=\mathbf{A}(j) \mathbf{h}(j)+\mathbf{n}(j) \in \mathbb{C}^{N}$, where $\mathbf{n}(j) \sim \mathcal{C N}(\mathbf{0}, \mathbf{C})$ and $\mathbf{C}=N_{0} \mathbf{I}_{N}$. The channel estimate based on the previous received observations $\hat{\mathbf{h}}(j \mid j)=\mathrm{E}[\mathbf{h}(j) \mid \mathbf{r}(0), \mathbf{r}(1), \cdots, \mathbf{r}(j)]$ can be obtained with the following Kalman recursive estimation procedure [5]

$$
\begin{aligned}
& \hat{\mathbf{h}}(j \mid j-1)=\mathbf{F} \hat{\mathbf{h}}(j-1 \mid j-1) \\
& \mathbf{M}(j \mid j-1)=\mathbf{F M}(j-1 \mid j-1) \mathbf{F}^{T}+\mathbf{Q} \\
& \mathbf{K}(j)=\mathbf{M}(j \mid j-1) \mathbf{A}(j)^{T}\left[\mathbf{C}+\mathbf{A}(j) \mathbf{M}(j \mid j-1) \mathbf{A}(j)^{T}\right]^{-1} \\
& \hat{\mathbf{h}}(j \mid j)=\hat{\mathbf{h}}(j \mid j-1)+\mathbf{K}(j)[\mathbf{r}(j)-\mathbf{A}(j) \hat{\mathbf{h}}(j \mid j-1)] \\
& \mathbf{M}(j \mid j)=(\mathbf{I}-\mathbf{K}(j) \mathbf{A}(j)) \mathbf{M}(j \mid j-1)
\end{aligned}
$$

In our experiments, the recursion is initialized by $\hat{\mathbf{h}}(-1 \mid-$ $1)=\mathbf{0}$ and $\mathbf{M}(-1 \mid-1)=100 \mathbf{I}$, reflecting little knowledge of the initial stage. From (5), we see that the inversion of an $N \times N$ matrix is required to find the Kalman gain $\mathbf{K}(j)$.

The Kalman filter employed above is based on the first order AR model for the fading process. Further improvement in the Kalman channel estimator will be possible with increased complexity if a higher order AR model is used. For instance, the correlation between channel coefficients can be more closely approximated by

$$
\mathbf{h}(j)=\mathbf{F}_{1} \mathbf{h}(j-1)+\mathbf{F}_{2} \mathbf{h}(j-2)+\mathbf{u}(j)
$$

leading to the second-order vector Gauss-Markov model and measurement model

$$
\begin{gathered}
{\left[\begin{array}{c}
\mathbf{h}(j-1) \\
\mathbf{h}(j)
\end{array}\right]=\left[\begin{array}{cc}
\mathbf{0} & \mathbf{I} \\
\mathbf{F}_{2} & \mathbf{F}_{1}
\end{array}\right]\left[\begin{array}{c}
\mathbf{h}(j-2) \\
\mathbf{h}(j-1)
\end{array}\right]+\left[\begin{array}{c}
\mathbf{0} \\
\mathbf{u}(j)
\end{array}\right]} \\
{\left[\begin{array}{c}
\mathbf{r}(j-1) \\
\mathbf{r}(j)
\end{array}\right]=\left[\begin{array}{cc}
\mathbf{A}(j-1) & \mathbf{0} \\
\mathbf{0} & \mathbf{A}(j)
\end{array}\right]\left[\begin{array}{c}
\mathbf{h}(j-1) \\
\mathbf{h}(j)
\end{array}\right]+\left[\begin{array}{c}
\mathbf{n}(j-1) \\
\mathbf{n}(j)
\end{array}\right]}
\end{gathered}
$$

For simplicity, we assume all the paths have equal received power and Doppler frequency in the following derivation. However, the extension to unequal power and Doppler frequency is straightforward. Now, the covariance matrix $\mathbf{Q}=\left[\begin{array}{cc}\mathbf{0} & \mathbf{0} \\ \mathbf{0} & \sigma_{Q}^{2} \mathbf{I}\end{array}\right]$ and the state of the system at the previous symbol time is $\left[\begin{array}{l}\mathbf{h}(j-2) \\ \mathbf{h}(j-1)\end{array}\right]$ and the state transition matrix $\mathbf{F}=\left[\begin{array}{cc}\mathbf{0} & \mathbf{I} \\ \mathbf{F}_{2} & \mathbf{F}_{1}\end{array}\right]$. The covariance matrix of noise vector $\left[\begin{array}{c}\mathbf{n}(j-1) \\ \mathbf{n}(j)\end{array}\right]$ becomes $\mathbf{C}=N_{0} \mathbf{I}_{2 N}$. Since different paths are uncorrelated with each other, we can assume $\mathbf{F}_{1}=f_{1} \mathbf{I}, \mathbf{F}_{2}=f_{2} \mathbf{I}$, and $f_{1}, f_{2}, \sigma_{Q}^{2}$ can be derived by the relationship $h_{k, l}[j]=f_{1} h_{k, l}[j-1]+f_{2} h_{k, l}[j-2]+u_{k, l}[j]$.

The procedure stated in (5) also applies to the second order Kalman filter with the following replacements:

$$
\begin{aligned}
& \mathbf{A}(j) \Longrightarrow\left[\begin{array}{cc}
\mathbf{A}(j-1) & \mathbf{0} \\
\mathbf{0} & \mathbf{A}(j)
\end{array}\right] ; \quad \mathbf{F} \Longrightarrow\left[\begin{array}{cc}
\mathbf{0} & \mathbf{I}_{N} \\
\mathbf{F}_{2} & \mathbf{F}_{1}
\end{array}\right] \\
& \mathbf{Q} \Longrightarrow\left[\begin{array}{cc}
\mathbf{0} & \mathbf{0} \\
\mathbf{0} & \sigma_{Q}^{2} \mathbf{I}
\end{array}\right] ; \quad \mathbf{h}(j) \Longrightarrow\left[\begin{array}{c}
\mathbf{h}(j-1) \\
\mathbf{h}(j)
\end{array}\right]
\end{aligned}
$$

The complexity of the second order Kalman filter increases compared to the first order due to the inversion of a $2 N \times 2 N\left(n N \times n N\right.$ for the $n^{t h}$ order Kalman filter $)$ matrix required to compute the Kalman gain.

In addition to estimation of time varying multipath coefficients, it was shown in [7] that the extended Kalman filter (EKF) can be used to estimate code delay. In this case, the observation (measurement) sequence is nonlinear in the state variables (the propagation delays), the ordinary Kalman filter equations cannot be used. Thus the observation sequence must be linearized to allow for a recursive estimation procedure using the Kalman filter equations. The EKF is a practical solution to this problem. It utilizes a Taylor's series approximation to obtain a linearized measurement sequence, at which point the ordinary filter equations can be employed.

\section{Numerical Results}

In our simulations, each user transmits one of $M=8$ Walsh codewords spread to a total length of $N=64$ chips. The effective spreading of the system is $N / \log _{2} M=64 / 3$ chips per bit. Different users are separated by different scrambling codes $\mathbf{C}_{k}(j)$ which are random, and differ from symbol to symbol.

For simplicity, the simulated system is assumed to be chip-synchronous, i.e., all path delays are assumed to be multiples of $T_{c}$. However, the system is asynchronous on the symbol level. Perfect slow power control is assumed in the sense that $P_{k}=\sum_{l=1}^{L_{k}} P_{k, l}$, the average received power, is equal for all users. The channel coefficients are normalized so that the channel for each user has unity gain. That is the coefficients satisfy $P_{k}=\sum_{l=1}^{L_{k}} P_{k, l}=1$. The system was simulated for 2-path channels, $L_{k}=L=2$ for all $k$. 


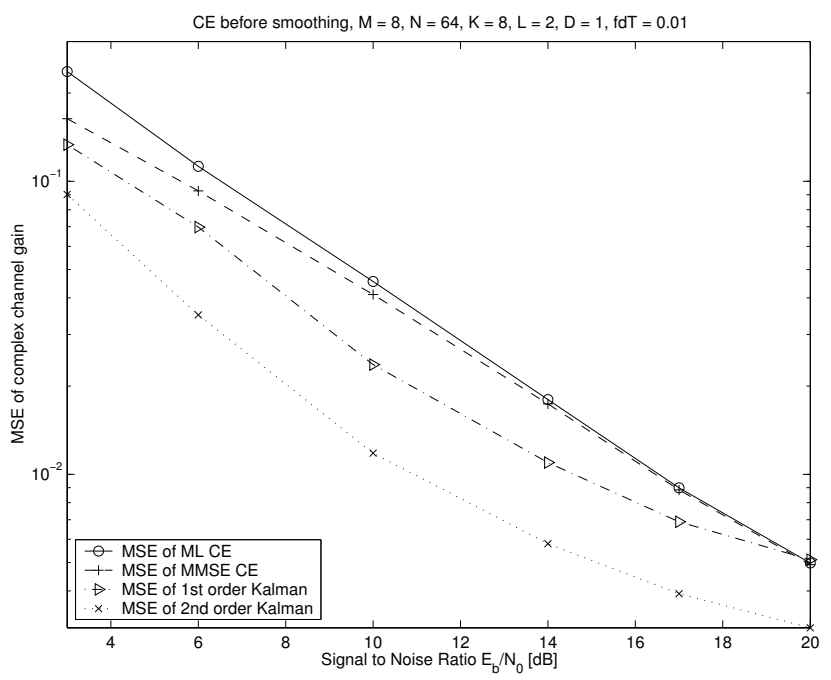

(a) MSE of CE vs. SNR.

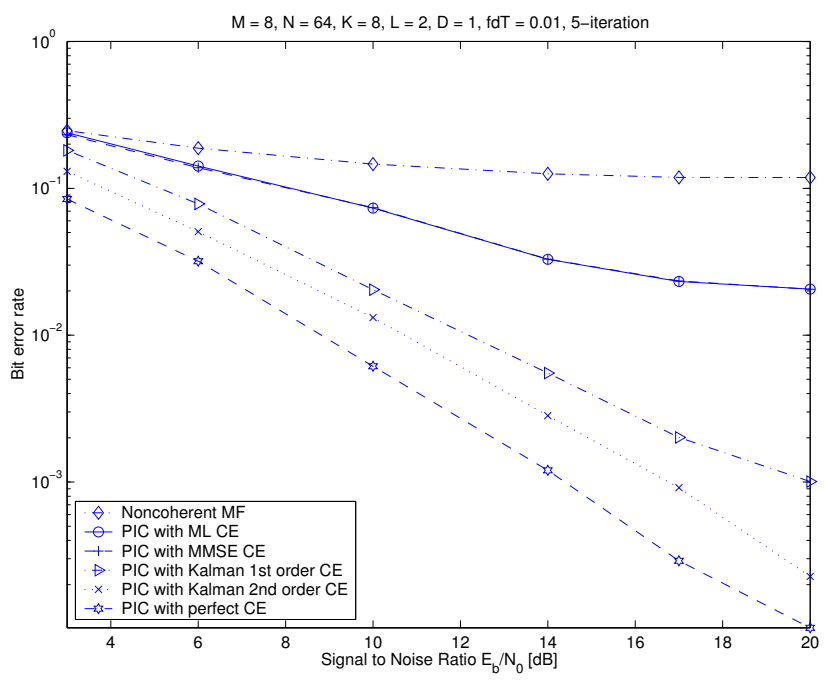

(b) BER vs. SNR.

Fig. 2. Comparison of different channel estimators with 5-stage PIC without smoothing.

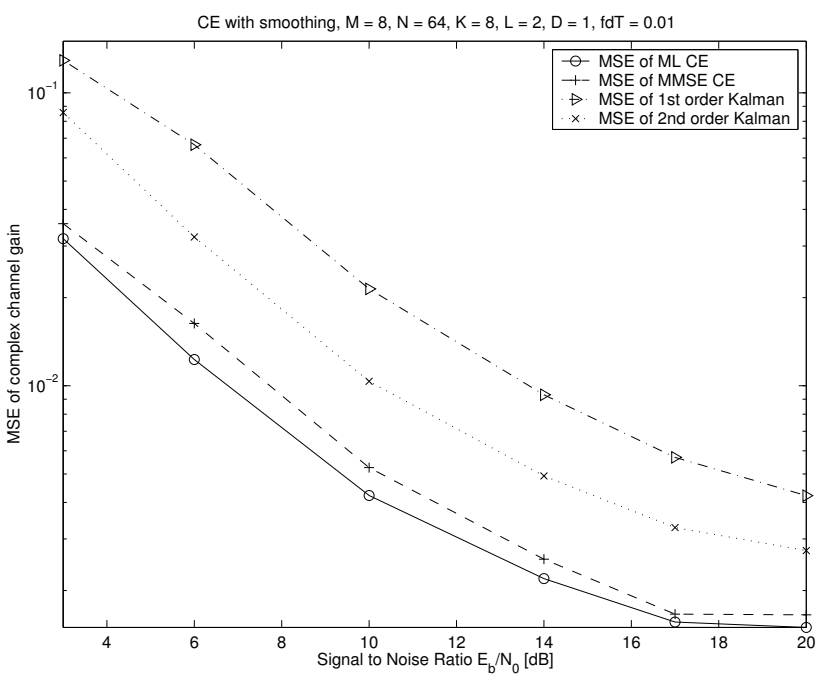

(a) MSE of CE vs. SNR.

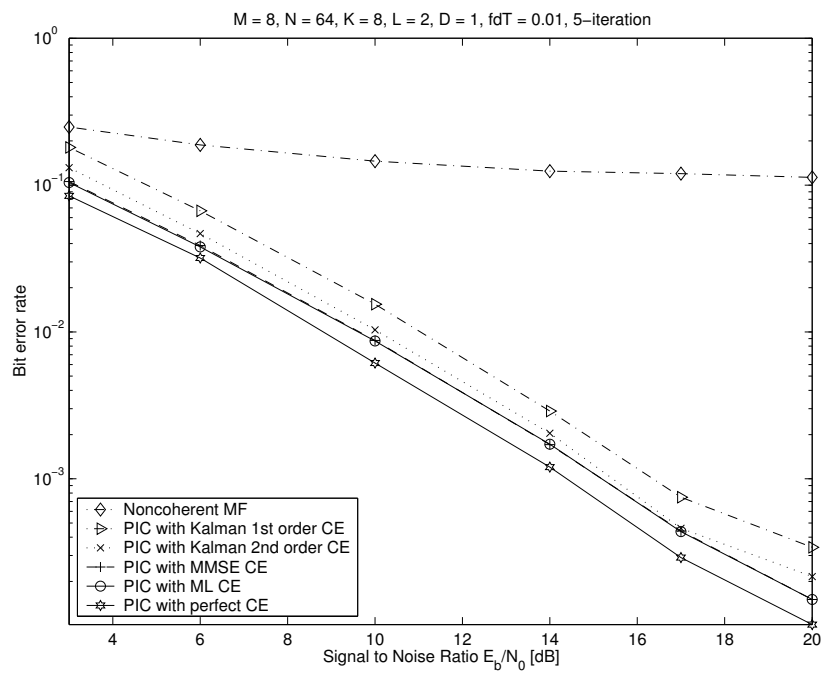

(b) BER vs. SNR.

Fig. 3. Comparison of different channel estimators with 5-stage PIC with smoothing.

The number of users is $K=8$. The simulation results are averaged over random distributions of fading, noise, delay, and scrambling code through numerous Monte-Carlo runs.

Coupled with 5-stage PIC, different channel estimators without the smoothing are assessed and compared in Fig. 2 in terms of estimation MSE and BER performance. The normalized Doppler frequency is set to $f_{d} T=0.01$ here. As expected, the ML algorithm has the worst performance. The LMMSE estimator considers the noise effect and slightly improves the estimation results, especially in the low SNR region (the BER performance remains the same though). The Kalman filters take advantage of correlative nature of the fading channel and significantly improve the estimation results and BER performance. The PIC is also simulated with perfect channel estimates, i.e., when $\hat{\mathbf{h}}_{k}=\mathbf{h}_{k}$ (which is called genie-aided PIC) to see how close the performance of proposed channel estimators is to the ideal one. It is also evident from the figures that PIC with the second order Kalman filter has the closest performance to genie-aided case. It outperforms the first order Kalman filter with the penalty of higher computational complexity.

Different channel estimators combined with the smooth- 


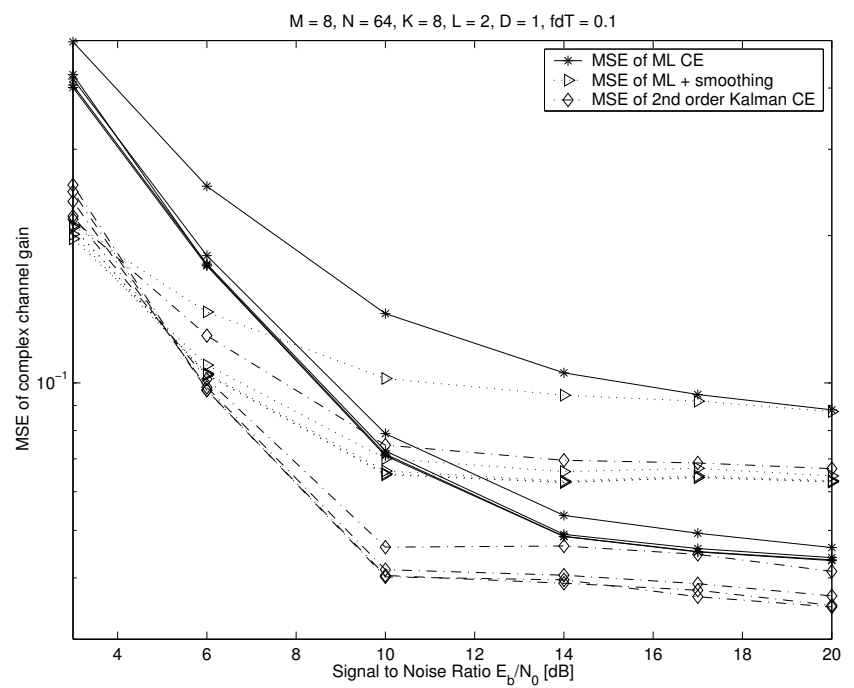

Fig. 4. ML vs. Kalman CE with 5-stage PIC in fast fading channels. The topmost curve represents the first stage estimation, the bottommost curve represents the last (the $5^{\text {th }}$ ) stage estimation.

ing filter are compared in Fig. 3. One can observe that the ML algorithm yields the smallest MSE and lowest BER after smoothing. The estimation MSE of the LMMSE algorithm is a little higher, but the BER performance is essentially the same. The ML is preferable to the LMMSE in that it does not need the knowledge of noise variance and the average received power of each signal path. No significant improvement is observed for the two Kalman filters after smoothing. Since the Kalman algorithm itself already takes the correlation into account, the additional smoothing does not make much difference. The ML estimator with channel smoothing appears to be the most favorable choice for estimating slow fading channels.

Based on the above experimental results, we come to important conclusions that the knowledge of the channel is crucial to the system performance and the quality of the channel estimation directly translates into the performance of data detection.

The ML estimator and Kalman filter are compared in the fast fading channels in Fig. 4 when the normalized Doppler frequency $f_{d} T=0.1$. The smoothing filter length is chosen to be 9 in this case. The figure shows that the second order Kalman filter outperforms the ML estimator under such circumstances. The latter can not keep track on the fast time-varying channel. The additional filtering operation can have opposite effect, it may destroy the details of the channel information. Fig. 4 shows that the ML estimator with smoothing yields higher estimation variance. The Kalman filter, on the contrary, is capable of tracking the fast fading channels, and achieves lower estimation error as well as better BER performance compared to the ML estimator. (The BER plot is not shown to conserve space).

\section{Conclusions}

In this paper, some channel estimation algorithms are presented for the DS-CDMA systems employing $M$-ary orthogonal modulation and long spreading codes over multipath fading channels. We focus on the decision directed approach without training sequences.

It is shown that ML estimator is efficient when the channel characteristic is unknown, and that when coupled with PIC, decision directed ML estimator with an additional smoothing filter performs better than the Kalman filters in slow fading channels. However, the filtering process causes delay for channel estimation which is not desirable under some circumstances. Also the ML estimator is subject to dimensionality problem when the number of users and paths increase. This problem can be tackled by stacking the received vectors provided the channel remains static during several symbol intervals. When this assumption is not valid, we can resort to the Kalman filter which is suitable for tracking fast fading channels. It takes both correlation and noise into account, does not have dimensionality problem and does not require an additional smoothing operation.

\section{REFERENCES}

[1] E. Ström, S. Miller. "Iterative demodulation and channel estimation of orthogonal signalling formats in asynchronous DS-CDMA systems". IEICE Transactions on Electronics, vol. E85-C, no. 3, pp. 442-451, March, 2002.

[2] J. Kim, S. Yoon, S. Kang, C. Kang. "A channel estimation technique in a DS/CDMA system with noncoherent M-ary orthogonal modulation in multipath fading channels". Proc. VTC'98, vol. 3, pp. 2383-2387, May 1998.

[3] J. Kim, S. Yoon, S. Kang, C. Kang. "Interference cancellation technique using channel parameter estimation in DS/CDMA system with M-ary orthogonal modulation." Electronic Letters, vol. 34, no. 12 , pp. 1194-1195, June 1998.

[4] S. Gollamudi, S. Ngaraj, Y. Huang, R. Buehrer. "Optimal multistage interference cancellation for CDMA systems using the nonlinear MMSE criterion." Proc. the 32nd Asilomar Conference on Signals, Systems, and Computers, pp. 665-669, Nov. 1998.

[5] S. Kay. "Fundamentals of statistical signal processing". Prentice Hall, NJ, 1998.

[6] M. Tsatsanis, G. Giannakis, G. Zhou. "Estimation and equalization of fading channels with random coefficients". Proc. ICASSP, vol. 2, pp. 1093-1096, May 1996.

[7] R. Iltis. "Joint estimation of PN code delay and multipath using the extended Kalman filter". IEEE Transactions on Communications, vol. 38, no. 10, pp. 1677-1685, Oct. 1990.

[8] R. Iltis, A. Fuxjaeger. "A digital DS spread-spectrum receiver with joint channel and doppler shift estimation". IEEE Transactions on Communications, vol. 39, no. 8, pp. 1255-1267, August 1991.

[9] K. Shanmugan, M. Sanchez, L. Haro, M. Calvo. "Channel estimation for $3 \mathrm{G}$ wideband CDMA systems using the Kalman filtering algorithm". IEEE International Conference on Personal Wireless Communications, pp. 95-97, Dec. 2000. 